\title{
Capacidad de peso para edad y longitud para edad para predecir desmedro a los tres años de vida*
}

Juan Rivera, M. Sc., Ph. D., ${ }^{(1)}$ Cecilia Cortés, Lic. en N utr., ${ }^{(1)}$ Mario Flores, M.C., ${ }^{(1)}$

Teresa González-C ossío, M. Sc., Ph. D. ${ }^{(1)}$

Rivera J, Cortés C, Flores M, González-Cossío T. Capacidad de peso para edad y longitud para edad para predecir desmedro a los tres años de vida. Salud Publica Mex 1998;40:127-132.

\section{Resumen}

Objetivo. Comparar la capacidad de dos valores de demarcación de peso para la edad y de longitud para la edad (-1 y -2 desviaciones estándar) a diferentes edades, en un intervalo que va del nacimiento a los 30 meses, para predecir desmedro a los tres años de edad. Material y métodos Utilizando datos de un estudio longitudinal en G uatemala, se evaluó la hipótesis de que la capacidad de distintos valores de demarcación de peso para edad y longitud para edad para predecir desmedro a los tres años de vida varía en función de la edad. Resultados. La longitud para edad a -1 desviación estándar (DE) del patrón de referencia O MS/ NCHS es un indicador pronóstico satisfactorio durante el primer semestre de vida, mientras que el mismo índice a -2 DE es satisfactorio despúes de los nueve meses. En cambio, peso para edad a -1 DE es un buen indicador exclusivamente a los nueve meses, mientras que entre los 15 y los 24 meses, este valor se comporta adecuadamente. Conclusión. La capacidad para predecir desmedro a los tres años de edad de distintos valores de demarcación de peso para edad y longitud para edad varía en función de la edad.

Palabras clave: desnutrición; desmedro; indicadores antropométricos; crecimiento
Rivera J, Cortés C, Flores M, González-Cossío T.

Prediction capacity of weight-for-age and length-for-age for risk of stunting in

three year old children.

Salud Publica Mex 1998;40:127-132.

\begin{abstract}
A bstract
Objective. To compare the capacity of two cut-off points of weight-for-age and length-for-age (-1 and -2 standard deviations) at different ages in the interval between birth and 30 months to predict stunting in three year old children. Material and methods $D$ ata from a longitudinal study in Guatemala were used to evaluate the hypothesis that the capacity of cut-off values of weight-for-age and length-forage to predict stunting at three years of age varies according to age. Results. Length-for-age at - 1 standard deviation (SD) of the W HO/N CHS mean reference values is a satisfactory prognostic indicator of stunting at three years of age during the first semester of life, while the same index at -2 SD is adequate after 9 months of age. Weight-forage at -1 SD is a suitable indicator only at 9 months and at -2 SD the indicator is satisfactory between 15 and 24 months of age. Conclusion. The capacity to predict stunting at three years of age, for weight-for-age and length-forage cut-off values varies according to age.
\end{abstract}

Key words: malnutrition; stunting; anthropometric indicators; growth

\footnotetext{
* Las tareas de análisis de datos fueron financiadas en parte con subvenciones de los Institutos N acionales de Salud de Estados Unidos (R01 HDD22440-07A1) y de la fundación PEW Charitable Trust 90-00210-00.

(1) Dirección de N utrición y Salud, Centro de Investigación en Salud Poblacional, Instituto N acional de Salud Pública, México.
}

Fecha de recibido: 30 de junio de 1997 • Fecha de aprobado: 28 de enero de 1998

Solicitud de sobretiros: Dr. Juan Rivera Dommarco. Dirección de N utrición y Salud. Centro de Investigación en Salud Poblacional. Instituto N acional de Salud Pública. Avenida Universidad 655, colonia Santa María A huacatitlán, 62508 Cuernavaca, Morelos, México. Correo electrónico: jrivera@ insp3.insp.mx 
E l desmedro (retardo en talla) es la manifestación predominante de crecimiento inadecuado en niños menores de cinco años en el mundo. En América Latina la prevalencia de desmedro fluctúa entre 16.5 y 56.7\%. ${ }^{1}$ Según la Encuesta Nacional de Nutrición, la prevalencia de desmedro severo, esto es, talla para la edad $<-2$ desviaciones estándar (DE) del patrón de referencia de la Organización Mundial de la Salud/Centro Nacional de Estadística en Salud (OMS/ $\mathrm{NCHS}){ }^{2}$ en menores de cinco años en México es de $23 \%$, mientras que la prevalencia de emaciación, es decir, peso para la talla $<-2 \mathrm{DE}$ del mismo patrón de referencia, es de sólo 6\%. ${ }^{3}$ Esto significa que también en México el desmedro es el principal problema de retardo en crecimiento y se debe a la interacción entre desnutrición e infección. ${ }^{4,5}$ El desmedro severo a los tres años de vida se asocia con niveles de salud y de desempeño físico e intelectual más bajos en niños, adolescentes y adultos. Tanto el desmedro como sus efectos adversos asociados se atenúan cuando mejora la alimentación de los niños durante los primeros dos a tres años de vida. ${ }^{6-9}$

Aunque el peso para edad es el indicador antropométrico de mayor uso para identificar a posibles beneficiarios de programas de alimentación y nutrición, la talla para edad es un indicador superior para identificar oportunamente a los niños con riesgo de desmedro. ${ }^{10}$ Sin embargo, cualquiera que sea el indicador utilizado, habitualmente se emplea un solo valor de demarcación para identificar a los beneficiarios de ese tipo de programas, independientemente de la edad del niño. Existen razones para suponer que la capacidad de diferentes valores de demarcación para identificar riesgo de desmedro varía con la edad. Debido a que el retardo en el crecimiento es un proceso cumulativo, es natural suponer que pequeñas desviaciones del crecimiento a edades tempranas pueden relacionarse con grandes déficits de talla a los tres años de vida, mientras que desviaciones del crecimiento similares en términos de la distribución de los valores de referencia, en edades posteriores, cercanas a los tres años, pueden no relacionarse con grandes déficits a esa edad. En este trabajo se compara la capacidad de dos valores de demarcación de peso para la edad y de talla para la edad (-1 y -2 DE) a diferentes edades, dentro de un intervalo que va del nacimiento a los 30 meses, para predecir desmedro a los tres años de edad. La predicción de desmedro a los tres años de edad es importante porque el proceso de retardo en estatura sucede, primordialmente, antes de esa edad, y las in- tervenciones nutricionales tienen poco o nulo impacto en el crecimiento después de los tres años de vida. ${ }^{9}$

La capacidad de un indicador para identificar adecuadamente a individuos que presentan un problema de salud determinado, en este caso niños con desmedro, se evalúa a partir de la sensibilidad, la especificidad y el valor predictivo positivo (VPP). La sensibilidad, para fines de este estudio, se define como la capacidad del indicador evaluado para identificar correctamente a los niños con desmedro a los tres años de vida, momento en que los efectos de una inadecuada nutrición son claramente evidentes. La especificidad es la capacidad del indicador para identificar correctamente a los niños sin desmedro. El VPP es la proporción de los niños seleccionados por el indicador que efectivamente presentan desmedro a los tres años.

\section{Material y métodos}

Los datos se obtuvieron de un estudio longitudinal de crecimiento y desarrollo que condujo el Instituto de Nutrición de Centroamérica y Panamá (INCAP), entre 1969 y 1977, en cuatro comunidades rurales ubicadas en el oriente de Guatemala. La prevalencia de desmedro en la población estudiada era muy alta; por ejemplo, se encontró que $62 \%$ de los niños de 36 meses de edad tenían valores inferiores a $-2 \mathrm{DE}$ del patrón de referencia OMS/NCHS. Se seleccionaron dos comunidades en forma aleatoria y se les administró un complemento alimenticio en forma de bebida, alto en contenido de energía y proteínas (Atole), mientras que las otras dos comunidades recibieron una bebida baja en energía y sin proteínas (Fresco). La descripción detallada del estudio puede consultarse en otras publicaciones. ${ }^{11}$ La muestra que se utilizó para el presente análisis incluyó a los niños que tenían datos completos de peso y longitud en decúbito supino en los siguientes puntos, entre los 3 y los 36 meses de edad: $3,6,9,12,15,18,21,24,30$ y 36 meses ( $n=302)$.

El peso se obtuvo mediante básculas pesa-bebé de palanca, con precisión de $10 \mathrm{~g}$. La longitud en decúbito se midió hasta el milímetro más cercano con estadímetros de madera. Para ambas medidas se utilizaron técnicas aceptadas internacionalmente. ${ }^{12}$ Los antropometristas fueron entrenados y estandarizados en la obtención de peso y longitud utilizando la técnica de Habicht. ${ }^{13}$

La variable dependiente fue la estatura para edad a los 36 meses de edad, con la que se generó una variable dicotómica utilizando como valor de demar- 
cación -2 DE del patrón de referencia de OMS/NCHS. ${ }^{2}$ Los niños con estaturas inferiores a este valor fueron clasificados como niños con desmedro.

Las variables independientes fueron los índices antropométricos del peso para edad y la longitud para edad (denominada en adelante como talla para edad), a las diferentes edades estudiadas entre los 3 y los 30 meses de vida. Se evaluaron dos valores de demarcación para cada índice antropométrico y cada edad: -1 y -2 DE de la distribución de referencia OMS/ NCHS. ${ }^{2}$

Para cada índice antropométrico, valor de demarcación y edad se obtuvieron las estimaciones de riesgo de presentar desmedro a los tres años mediante regresión logística ${ }^{14}$ y se calculó la sensibilidad, la especificidad y el valor predictivo positivo. Los análisis se realizaron utilizando el paquete estadístico STATA. ${ }^{15}$

\section{Resultados}

El cuadro I presenta información para el índice peso para edad, y el cuadro II, información para el índice talla para edad, con valores de sensibilidad y especificidad, y valor predictivo para los dos valores de demarcación (-1 y -2 DE).

La figura 1 presenta valores de sensibilidad, y la figura 2, valores de especificidad para los dos índices antropométricos con los dos valores de demarcación.

Los valores de sensibilidad para el indicador talla para edad a -1 DE son superiores a 70\% a lo largo de todas las edades, mientras que para el indicador talla para edad a -2 DE los valores de sensibilidad son de $70 \%$ o menos durante los primeros nuevemesesde vi-

\section{Cuadro I}

SensibILIDAd, especificidad Y VPP del índice Peso PARA EDAD A VALORES DE DEMARCACIÓN DE -1 Y -2 DE EN RELACIÓN CON DESMEDRO A LOS TRES AÑOS DE EDAD

EDAD Peso para edad <-1 DE Peso para edad <-2 DE (meses) Sensibilidad Especificidad VPP Sensibilidad Especificidad VPP

\begin{tabular}{rrrrrrr}
3 & 30.3 & 87.8 & 80.3 & 4.8 & 99.1 & 90.0 \\
\hline 6 & 56.9 & 74.8 & 78.7 & 16.5 & 95.7 & 86.1 \\
\hline 9 & 85.6 & 40.0 & 70.0 & 46.3 & 88.7 & 87.0 \\
\hline 12 & 92.6 & 22.6 & 66.2 & 63.3 & 70.4 & 77.8 \\
\hline 15 & 96.3 & 16.5 & 65.3 & 73.4 & 64.3 & 77.1 \\
\hline 18 & 96.8 & 18.3 & 65.9 & 74.5 & 71.3 & 80.9 \\
\hline 21 & 97.9 & 18.3 & 66.2 & 72.3 & 73.9 & 81.9 \\
\hline 24 & 97.3 & 25.2 & 68.0 & 69.7 & 81.7 & 86.2 \\
\hline 30 & 93.6 & 34.8 & 70.1 & 59.6 & 90.4 & 91.1
\end{tabular}
PARA EDAD A VALORES DE DEMARCACIÓN DE -1 Y -2 DE EN RELACIÓN CON DESMEDRO A LOS TRES AÑOS DE EDAD

EDAD Talla para edad <-1 DE Talla para edad < -2 DE (meses) Sensibilidad Especificidad VPP Sensibilidad Especificidad VPP

\begin{tabular}{rrrrrrr}
3 & 72.9 & 66.1 & 77.9 & 24.5 & 93.9 & 86.8 \\
\hline 6 & 89.4 & 38.3 & 70.3 & 50.5 & 90.4 & 89.6 \\
\hline 9 & 98.4 & 28.9 & 67.0 & 71.3 & 81.7 & 86.5 \\
\hline 12 & 99.5 & 15.7 & 65.9 & 88.8 & 67.8 & 81.9 \\
\hline 15 & 99.5 & 14.8 & 65.6 & 93.1 & 53.9 & 76.8 \\
\hline 18 & 100.0 & 0.0 & 64.6 & 97.3 & 50.4 & 76.3 \\
\hline 21 & 100.0 & 0.0 & 64.6 & 96.3 & 51.3 & 76.4 \\
\hline 24 & 100.0 & 0.0 & 63.9 & 94.7 & 71.3 & 84.4 \\
\hline 30 & 100.0 & 0.0 & 64.2 & 94.2 & 85.2 & 91.2
\end{tabular}

da y superiores a este valor de los 12 a los 30 meses (figura 1).

Los valores de sensibilidad para el indicador peso para edad a -1 DE son menores a 70\% durante los primeros seis meses de vida y superiores a este valor de los 9 a los 30 meses. En cambio, los valores de sensibilidad son generalmente bajos para el indicador peso para edad a -2 DE (figura 1).

Los valores de especificidad para el indicador talla para edad a -1 DE son superiores a $35 \%$ solamente durante el primer semestre de vida, para caer en edades posteriores en valores de hasta cero. En cambio, para el indicador talla para la edad a $-2 \mathrm{DE}$, los valores de especificidad son superiores a $50 \%$ a lo largo de todas las edades (figura 2).

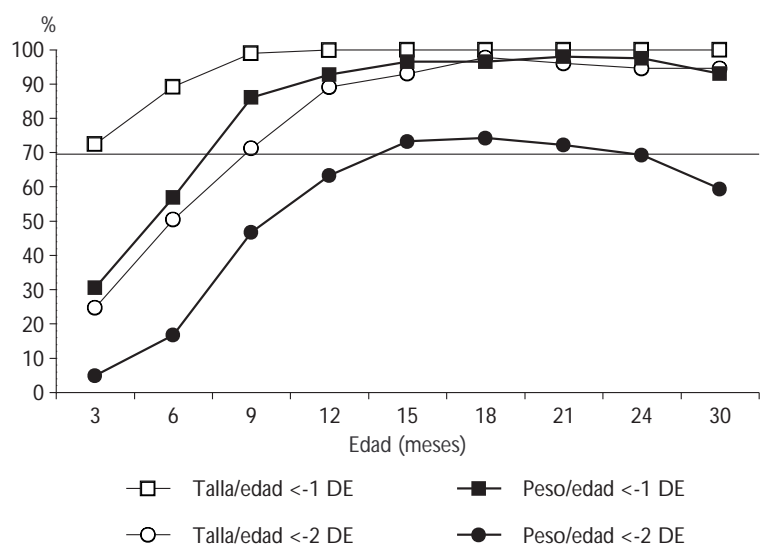

Figura 1.Sensibilidad de talla para edad y peso para EDAD CON VALORES DE DEMARCACIÓN DE -1 DE Y -2 DE A DISTINTAS EDADES 
Los valores de especificidad para el indicador peso para edad a $-1 \mathrm{DE}$ son superiores a $35 \%$ únicamente durante los primeros nueve meses de vida. En edades posteriores estos valores se mantienen por debajo de dicho valor. Para el indicador peso para edad a -2 DE los valores de especificidad son superiores a $60 \%$ a lo largo de todas las edades (figura 2).

\section{Discusión}

Los valores aceptables de los indicadores de capacidad predictiva (sensibilidad, especificidad y valor predictivo positivo) deben definirse a la luz de la importancia que, desde la perspectiva de la salud pública, reviste el problema que se estudia (su magnitud, relevancia y efectos sociales) y a partir del costo, la efectividad y la inocuidad de las intervenciones disponibles para prevenirlo o controlarlo. En este caso, el desmedro a los tres años de vida es un importante problema de salud pública no sólo en México sino en todo el orbe, como lo muestran los datos de la introducción. Su relevancia radica en los efectos adversos sobre la salud y la probabilidad de sobrevivencia durante la niñez, así como sobre el desempeño físico e intelectual durante la adolescencia y la edad adulta. ${ }^{6-8}$ Existen intervenciones de probada efectividad y de relativo bajo costo para prevenir el desmedro. Tal es el caso de las estrategias de comunicación educativa dirigidas a mejorar la alimentación infantil, la promoción de la lactancia y la suplementación alimentaria, entre otras. Esta última ha resultado de gran efectividad en la reducción del retardo en crecimiento ${ }^{16,17}$ a bajo costo $^{18}$ y sin efectos adversos en los beneficiarios. La razón de

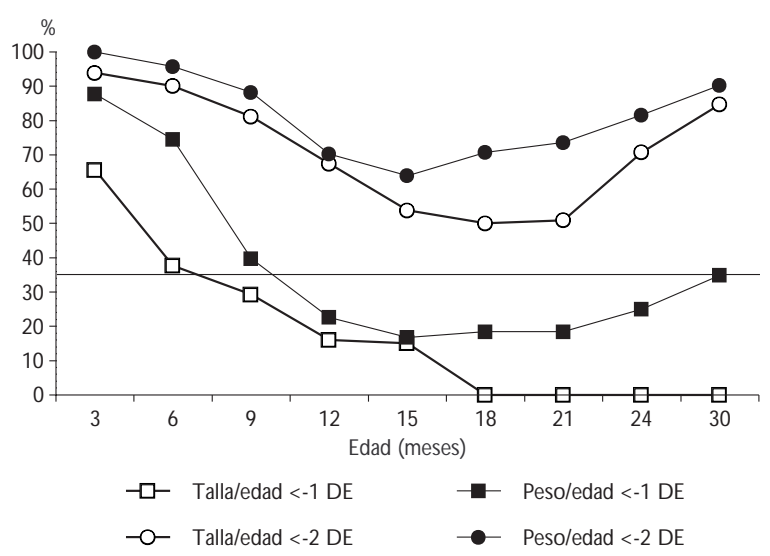

Figura 2. EsPeCIFICIDAD DE TALLA PARA EDAD Y PESO PARA EDAD CON VALORES DE DEMARCACIÓN DE -1 DE Y -2 DE A DISTINTAS EDADES este bajo costo es que se ha demostrado que con cantidades muy pequeñas de alimentación complementaria de buena calidad (entre 100 y $200 \mathrm{kcal}$ ) se logran efectos positivos en el crecimiento lineal. ${ }^{16-17}$ En consideración a lo anterior, al evaluar los indicadores para la predicción de desmedro se ha privilegiado la sensibilidad sobre la especificidad y el VPP, teniendo cuidado de mantener valores de especificidad y VPP razonables. Así, se juzgaron favorablemente los indicadores con sensibilidad de $70 \%$ o más, VPP de $60 \%$ o más y especificidad superior a $35 \%$. La racionalidad de estos criterios se ilustra mediante el siguiente ejemplo: a los seis meses de edad el indicador talla para edad a -1 DE presentó los siguientes valores: sensibilidad $=89 \%$, especificidad $=38 \%$ y VPP $=70 \%$. El significado de estos valores es el siguiente: el indicador permite identificar a $89 \%$ de los niños que eventualmente presentarán desmedro a los 36 meses, de tal manera que pueden incluirse en las intervenciones efectivas para prevenir desmedro. Del total de niños seleccionados por el indicador, $70 \%$ requiere la intervención, es decir, solamente $30 \%$ la recibirá sin necesitarla, lo que es razonable dado el bajo costo de las intervenciones en nutrición.

Los VPP para los cuatro indicadores evaluados son superiores a $60 \%$ para todas las edades, y se consideran satisfactorios dado el bajo costo de las acciones nutrimentales.

Atendiendo exclusivamente a los valores de sensibilidad, el mejor indicador pronóstico de desmedro a los tres años es el de la talla para edad a $-1 \mathrm{DE}$, seguido del peso para edad a $-1 \mathrm{DE}$ y de la talla para edad a $-2 \mathrm{DE}$, los cuales tienen valores muy similares. El indicador con menores valores de sensibilidad, considerados como insatisfactorios a todas las edades, es el de peso para edad a -2 DE (figura 1).

En contraste, los valores de especificidad de los indicadores a valores de demarcación de $-1 \mathrm{DE}$ son muy bajos en edades posteriores a los 9 o 12 meses, al extremo de que entre los 18 y los 30 meses la especificidad de la talla para edad es de cero, lo que se traduce en la selección de la totalidad de los niños estudiados; es decir, el indicador se torna totalmente inútil. En cambio, para valores de demarcación de -2 DE los valores de especificidad son altos a lo largo de todas las edades (figura 2).

Considerando simultáneamente los valores de sensibilidad y especificidad se concluye lo siguiente:

1. Talla para edad: el valor de demarcación de $-1 \mathrm{DE}$ se comporta favorablemente en los primeros seis meses de vida, mientras que en edades posteriores la especificidad adquiere valores sumamente 
bajos. En contaste, el valor de demarcación de -2 DE se comporta de manera desfavorable durante los primeros seis meses de vida, cuando los valores de sensibilidad son de $50 \%$ o menores, mientras que a partir de los 9 meses y hasta los 30 meses de vida, dicho indicador se comporta de manera favorable. Los resultados para los nueve meses de edad no son concluyentes, por lo que no es posible hacer recomendaciones sobre el punto de corte apropiado a esta edad. En resumen, para el índice talla para edad el valor de demarcación de $-1 \mathrm{DE}$ es superior durante los primeros seis meses de vida, mientras que $-2 \mathrm{DE}$ es superior a partir de los nueve meses.

2. Peso para edad: durante el primer semestre ninguno de los valores de demarcación se comporta satisfactoriamente dado que los valores de sensibilidad son inferiores a $60 \%$. El valor de demarcación de -1 DE se comporta favorablemente pero sólo en un breve lapso, a los nueve meses de edad, ya que en edades sucesivas la especificidad adquiere valores muy bajos. El valor de demarcación -2 DE presenta valores aceptables de sensibilidad entre los 15 y los 24 meses. En resumen, el índice peso para edad es poco útil antes de los nueve meses de vida. El valor de demarcación de -1 DE es aceptable y superior a $-2 \mathrm{DE}$ a los nueve meses, mientras que $-2 \mathrm{DE}$ es satisfactorio y superior a -1DE entre los 15 y los 24 meses.

Los resultados favorecen la hipótesis de este trabajo respecto a la diferencia en la capacidad para predecir desmedro a los tres años de vida que tienen distintos valores de demarcación de los indicadores peso para edad y talla para edad, a distintas edades del niño.

Dada la importancia de iniciar las intervenciones nutrimentales a edades muy tempranas, a fin de lograr un mayor impacto, ${ }^{9}$ se recomienda el uso de -1DE de talla para edad durante el primer semestre de vida para seleccionar a los beneficiarios de intervenciones en nutrición. Al respecto, una de las intervenciones durante el primer semestre de vida que ha tenido éxito es la promoción de prácticas adecuadas de lactancia. Sin duda, las conclusiones y recomendaciones son correctas para la población analizada; su relevancia para otras poblaciones depende fundamentalmente de la prevalencia de desmedro. Los valores de sensibilidad y especificidad son relativamente estables en poblaciones donde este problema de salud tiene diversas prevalencias. ${ }^{19}$ En cambio, el VPP es altamente dependiente de las diferencias en las prevalencias, ${ }^{19}$ de tal manera que es muy probable que la aplicación de estos hallazgos y de sus consiguientes recomendaciones resulte acertada en poblaciones con prevalencias de desmedro severo similares a las de la población estudiada (62\%) y que se observan en algunas zonas rurales marginadas de los estados del sur del país. Es necesario evaluar la relevancia de los hallazgos del presente estudio para poblaciones con prevalencias menores antes de recomendar su aplicación generalizada.

\section{Agradecimientos}

En el estudio longitudinal a partir del cual se elaboró este trabajo colaboraron investigadores, trabajadores de campo y personal del Centro de Cómputo del Instituto de Nutrición de Centroamérica y Panamá (IN$\mathrm{CAP})$, a quienes se agradece su valiosa participación.

\section{Referencias}

1. W orld Health O rganization. Expert Committee. Physical Status: The use and interpretation of anthropometry. W HO Technical Report Series 854. G inebra:1995;166-167.

2. 0 rganización Mundial de la Salud. Medición del cambio del estado nutricional. Ginebra:0 MS,1983.

3. Rivera J, González-C ossío T, Flores M, Hernández M, Lezana MA, Sepúlveda J. Emaciación y déficit de talla en menores de cinco años en distintas regiones y estratos en México. Salud Publica Mex 1995;37:95-107. 4. Lutter CK, Mora JO , Habicht JP, Rasmussen KM, Robson DS, Sellers SG et al. Nutritional supplementation: Effects on child stunting because of diarrhea. Am J Clin N utr 1989:50:1-8

5. Lutter C, Habicht JP, Rivera J, Martorell R. The relationship between energy intake and diarrheal disease in their effects on child growth: Biological model, evidence, and implications for public health policy. Food N utr Bull 1992;14(1):36-52.

6. Rivera JA, Martorell R, Ruel M, Habicht JP, Haas J. N utritional supplementation during the preschool years influences body size and composition of G uatemalan adolescents. J N utr 1995;125:1068S-1077S.

7. Pollitt E, Gorman K, Engle PL, Rivera JA, Martorell R. N utrition in early life and fulfillment of intellectual potential. J N utr 1995;125:1111S-1118S. 8. Haas J, Martínez E, Murdoch S, Conlisk E, Rivera JA, Martorell R. N utritional supplementation during the preschool years and physical work capacity in adolescent and young adult Guatemalans. J N utr 1995;125: 1078S-1089S.

9. Schroeder DG, Martorell R, Rivera JA, Ruel MT, Habicht JP. A ge differences in the impact of nutritional supplementation on growth. J N utr 1995;125:1051S-1059S.

10. Ruel M, Rivera J, Habicht JP. Length screens better than weight in stunted populations. J N utr 1995;125:1222-1228.

11. Habitch JP, Martorell R. 0 bjectives, research design and implementation of the IN CAP Iongitudinal study. Food N utr Bull 1992;14:176-190. 12. Lohman TG, Roche AF, Martorell R, ed. Anthropometric standardization reference manual. Champaign, Il.: Human Kinetics Publisher, 1988. 13. Habitch JP. Estandarización de métodos epidemiológicos cuantitativos sobre el terreno (Standardization of quantitative epidemiological methods in the field). Bull Pan Am Health O rgan 1976;76:375-384. 
14. Hosmer D, Lemeshow S. Applied logistic regression. N ueva York: W iley \& Sons, 1989.

15. Stata Corporation. Stata reference manual: Release 3.1. 6th edition. College Station, TX, 1993.

16. Mora JO, Herrera MG, Suescun J, de N avarro L,W agner M.The effect of nutritional supplementation on physical growth of children at risk of malnutrition. Am J C lin N utr 1995;34:1885-1892.

17. Habicht JP, Martorell R, Rivera JA. N utritional impact of supplementation in the IN C AP Longitudinal Study:A nalytic strategies and inferences. J N utr 1995;125:1042S-1050S.
18. Balachander J. Tamil nadu,s successful nutrition effort. En: Rhode J, Chatterjee M, Morley D, ed. Reaching health for all. Delhi: Oxford University Press, 1993.

19. Habicht JP. Some characteristics of indicators of nutritional status for use in screening and surveillance. Am J C lin N utr 1980;33:531-535. 